\title{
Erratum to: Radiology medical malpractice suits in gastrointestinal radiology: prevalence, causes, and outcomes
}

\author{
Stephen R. Baker • Shivam Shah • Shanchita Ghosh • \\ Alejandro Castro
}

Published online: 3 October 2014

(C) American Society of Emergency Radiology 2014

\section{Erratum to: Emerg Radiol}

DOI 10.1007/s10140-014-1268-3

The authors of this article would like to add Alejandro Castro as the final author. Mr. Castro was the statistician and they had forgotten to include him in the author listing.

In addition, Shivam Shah is now affiliated to both affiliations - he has been added on the first affiliation.

The online version of the original article can be found at http://dx.doi.org/ 10.1007/s10140-014-1268-3.

S. R. Baker · S. Shah · S. Ghosh · A. Castro

Department of Radiology, New Jersey Medical School-Rutgers

School of Biomedical and Health Sciences, Rutgers University,

Newark, NJ, USA

S. Shah $(\bowtie) \cdot$ S. Ghosh $\cdot$ A. Castro

Department of Medicine, New Jersey Medical School-Rutgers

School of Biomedical and Health Sciences Rutgers, The State

University of New Jersey, 185 South Orange Ave, Newark,

NJ 07103, USA

e-mail: shah.shivam@gmail.com 